\title{
Crystal structure of bis(3,3'-diethyl-4-maleimidophenyl)methane, $\mathrm{C}_{25} \mathrm{H}_{22} \mathrm{~N}_{2} \mathrm{O}_{4}$
}

\author{
H.-L. Zhu*,III, H. Zhang ${ }^{\mathrm{I}}$, J.-L. Ma', X.-Y. Qiu' ${ }^{\mathrm{I}}$ S. Yang ${ }^{\mathrm{I}}$ and L. Sun ${ }^{\mathrm{I}}$ \\ I Fuyang Normal College, Department of Chemistry, Fuyang Anhui 236041, P. R. China \\ II Wuhan University of Science and Engineering, Department of Chemistry, Wuhan, 430073, P. R. China
}

Received August 19, 2003, accepted and available on-line November 23, 2003; CCDC-No. 1267/1141

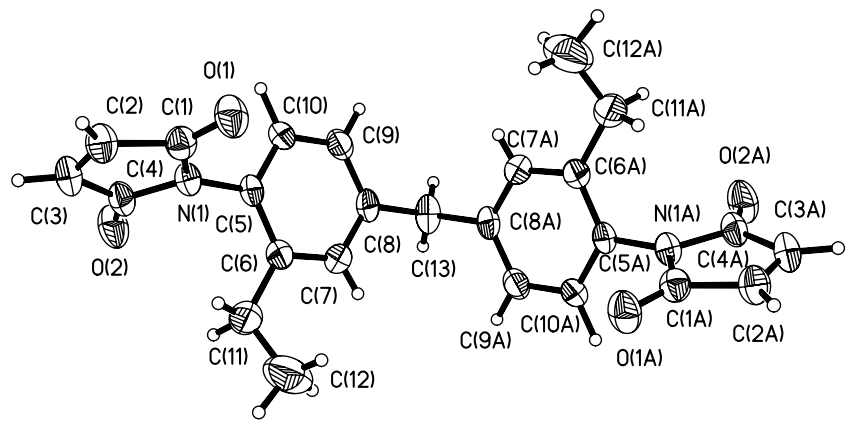

\begin{abstract}
$\mathrm{C}_{25} \mathrm{H}_{22} \mathrm{~N}_{2} \mathrm{O}_{4}$, orthorhombic, Pccn (No. 56), $a=12.745$ (2) $\AA$, $b=16.974(2) \AA, c=10.218(1) \AA, V=2210.4 \AA^{3}, Z=4$, $R_{\mathrm{gt}}(F)=0.114, w R_{\mathrm{ref}}\left(F^{2}\right)=0.300, T=293 \mathrm{~K}$.
\end{abstract}

\section{Source of material}

The title compound was prepared with the similar procedure to that used for bis(4-maleimidophenyl)methane [1]: An acetone solution $(15 \mathrm{ml})$ of maleic anhydride $(2 \mathrm{mmol}, 196 \mathrm{mg})$ and 3,3'-diethyl-4,4'-diaminophenylmethane ( $1 \mathrm{mmol}, 254 \mathrm{mg}$ ) was stirred for $1 \mathrm{~h}$, then acetic anhydride $(5 \mathrm{ml})$, anhydrous magnesium acetate $(0.2 \mathrm{~g})$ and triethylamine $(5 \mathrm{ml})$ were added. The resultant solution was heated to $353 \mathrm{~K}$ and allowed to stand for $1 \mathrm{~h}$. The solution was then cooled to room temperature. On addition of water, shiny yellow crystals were deposited, filtered and washed with water and acetone in turn. Recrystallization was carried out from ethanol; the crystals were dried in a vacuum desiccator over $\mathrm{CaCl}_{2}$ (yield 79\%). All reagents and solvents were used as obtained without further purification. $\mathrm{C}, \mathrm{H}$ and $\mathrm{N}$ elemental analyses were performed on a Perkin-Elmer analyzer. Composition calculated: C, 72.45\%; H, 5.35\%; N, 6.76\%; found: C, 71.98\%; H, $5.50 \% ; \mathrm{N}, 6.70 \%$.

\section{Experimental details}

The $\mathrm{H}$ atoms around $\mathrm{C}(11)$ and $\mathrm{C}(12)$ were positioned geometrically and treated as riding atoms, with $U_{\text {iso }}(\mathrm{H})=1.2 U_{\text {eq }}(\mathrm{C})$. All the other hydrogen atoms were refined isotropically. $\mathrm{C}(11)$ and $C(12)$ are disordered, however, we did not try to split them. Large values of $R$ factors were the results of fragile and very poor crystals and a data collection in a quite wide range $\left(2.8^{\circ} \leq \theta \leq 28.3^{\circ}\right)$.

\section{Discussion}

Recently we reported the structure of bis(4-maleimidophenyl)methane [1]. As a subsequent research, we report here the crystal structure diethyl-substituted bis(4-maleimidophenyl)methane. The asymmetric unit of the title complex contains one-half of the molecule, with the other half generated by a crystallographic twofold axis passing through the atom $\mathrm{C}(13)$. The bond lengths and angles of the phenylmaleimide moiety are comparable with those observed in the compound we reported previously [1]. The dihedral angle between the substituted phenyl and pyrrole rings is $71.6(2)^{\circ}$, which is much larger than that $\left(52.1(1)^{\circ}\right)$ in the similar bis(4-maleimidophenyl)methane [1]. This is mainly because that ethyl groups in the title compound has larger steric effects. Atoms $\mathrm{O}(1)$ and $\mathrm{O}(2)$ are coplanar with the pyrrole plane. The end-to-end distance of the molecule is 13.56(2) $\AA$, being close to that (13.54 $\AA$ ) in bis(4-maleimidophenyl)methane [1].

Table 1. Data collection and handling.
Crystal:

Wavelength:

$\mu$ :

Diffractometer, scan mode:

$2 \theta_{\max }$ :

$N(h k l)_{\text {measured }}, N(h k l)_{\text {unique: }}$ Criterion for $I_{\mathrm{obs}}, N(h k l)_{\mathrm{gt}}$ :

$N(\text { param })_{\text {refined: }}$

Program: yellow block, size $0.28 \times 0.38 \times 0.80 \mathrm{~mm}$

Mo $K_{\alpha}$ radiation $(0.71073 \AA)$

$0.85 \mathrm{~cm}^{-1}$

Siemens SMART CCD, $\omega$

$56.62^{\circ}$

12884,2738

$I_{\mathrm{obs}}>2 \sigma\left(I_{\mathrm{obs}}\right), 1725$

165

SHELXTL [2]
Table 2. Atomic coordinates and displacement parameters (in $\AA^{2}$ ).

\begin{tabular}{llllll}
\hline Atom & Site & $x$ & $y$ & $z$ & $U_{\text {iso }}$ \\
\hline $\mathrm{H}(11 \mathrm{~A})$ & $8 e$ & 0.0879 & 0.7319 & 0.2357 & 0.160 \\
$\mathrm{H}(11 \mathrm{~B})$ & $8 e$ & 0.1295 & 0.7613 & 0.1007 & 0.160 \\
$\mathrm{H}(12 \mathrm{~A})$ & $8 e$ & 0.1080 & 0.8610 & 0.2374 & 0.206 \\
$\mathrm{H}(12 \mathrm{~B})$ & $8 e$ & -0.0112 & 0.8404 & 0.2533 & 0.206 \\
$\mathrm{H}(12 \mathrm{C})$ & $8 e$ & 0.0316 & 0.8700 & 0.1181 & 0.206 \\
$\mathrm{H}(10)$ & $8 e$ & $-0.106(3)$ & $0.529(2)$ & $0.051(4)$ & $0.06(1)$ \\
$\mathrm{H}(7)$ & $8 e$ & $-0.094(3)$ & $0.796(3)$ & $0.008(4)$ & $0.07(1)$ \\
$\mathrm{H}(9)$ & $8 e$ & $-0.228(4)$ & $0.598(3)$ & $-0.076(4)$ & $0.09(1)$ \\
$\mathrm{H}(3)$ & $8 e$ & $0.265(4)$ & $0.510(3)$ & $0.270(5)$ & $0.10(2)$ \\
$\mathrm{H}(2)$ & $8 e$ & $0.124(4)$ & $0.499(2)$ & $0.437(5)$ & $0.08(1)$ \\
$\mathrm{H}(13)$ & $8 e$ & $-0.218(3)$ & $0.783(3)$ & $-0.197(5)$ & $0.09(1)$ \\
\hline
\end{tabular}

* Correspondence author (e-mail: hlzhu@wist.edu.cn) 
Table 3. Atomic coordinates and displacement parameters (in $\AA^{2}$ ).

\begin{tabular}{|c|c|c|c|c|c|c|c|c|c|c|}
\hline Atom & Site & $x$ & $y$ & $z$ & $U_{11}$ & $U_{22}$ & $U_{33}$ & $U_{12}$ & $U_{13}$ & $U_{23}$ \\
\hline $\mathrm{O}(1)$ & $8 e$ & $-0.0622(2)$ & $0.5595(2)$ & $0.3541(3)$ & $0.064(2)$ & $0.110(2)$ & $0.074(2)$ & $0.012(2)$ & $0.018(2)$ & $0.011(2)$ \\
\hline $\mathrm{O}(2)$ & $8 e$ & $0.1968(2)$ & $0.5950(2)$ & $0.0553(3)$ & $0.056(2)$ & $0.133(3)$ & $0.089(2)$ & $0.013(2)$ & $0.019(2)$ & $0.014(2)$ \\
\hline $\mathrm{N}(1)$ & $8 e$ & $0.0484(2)$ & $0.5911(2)$ & $0.1839(3)$ & $0.045(2)$ & $0.071(2)$ & $0.061(2)$ & $0.007(1)$ & $0.003(1)$ & $-0.002(2)$ \\
\hline $\mathrm{C}(1)$ & $8 e$ & $0.0235(3)$ & $0.5577(2)$ & $0.3049(4)$ & $0.060(2)$ & $0.067(2)$ & $0.060(2)$ & $0.004(2)$ & $-0.000(2)$ & $-0.007(2)$ \\
\hline$C(2)$ & $8 e$ & $0.1215(3)$ & $0.5216(3)$ & $0.3535(5)$ & $0.066(3)$ & $0.089(3)$ & $0.066(3)$ & $0.008(2)$ & $-0.010(2)$ & $0.003(2)$ \\
\hline$C(3)$ & $8 e$ & $0.1956(3)$ & $0.5317(3)$ & $0.2684(5)$ & $0.047(2)$ & $0.096(3)$ & $0.086(3)$ & $0.013(2)$ & $-0.010(2)$ & $0.000(2)$ \\
\hline$C(5)$ & $8 e$ & $-0.0245(2)$ & $0.6327(2)$ & $0.1017(3)$ & $0.038(2)$ & $0.066(2)$ & $0.058(2)$ & $0.008(2)$ & $0.005(1)$ & $-0.000(2)$ \\
\hline$C(6)$ & $8 e$ & $-0.0169(3)$ & $0.7134(2)$ & $0.0881(4)$ & $0.049(2)$ & $0.060(2)$ & $0.092(3)$ & $0.002(2)$ & $-0.007(2)$ & $-0.011(2)$ \\
\hline$C(7)$ & $8 e$ & $-0.0912(3)$ & $0.7485(3)$ & $0.0080(5)$ & $0.060(2)$ & $0.054(2)$ & $0.102(3)$ & $0.007(2)$ & $0.000(2)$ & $0.005(2)$ \\
\hline $\mathrm{C}(8)$ & $8 e$ & $-0.1701(3)$ & $0.7075(2)$ & $-0.0541(4)$ & $0.043(2)$ & $0.075(3)$ & $0.063(2)$ & $0.014(2)$ & $0.007(2)$ & $-0.006(2)$ \\
\hline $\mathrm{C}(9)$ & $8 e$ & $-0.1750(3)$ & $0.6273(3)$ & $-0.0348(4)$ & $0.044(2)$ & $0.074(3)$ & $0.076(3)$ & $-0.001(2)$ & $-0.001(2)$ & $-0.013(2)$ \\
\hline $\mathrm{C}(10)$ & $8 e$ & $-0.1020(3)$ & $0.5894(2)$ & $0.0426(4)$ & $0.043(2)$ & $0.060(2)$ & $0.081(3)$ & $-0.004(2)$ & $0.004(2)$ & $-0.002(2)$ \\
\hline$C(11)$ & $8 e$ & $0.0684(4)$ & $0.7606(3)$ & $0.1573(9)$ & $0.090(4)$ & $0.070(3)$ & $0.240(8)$ & $0.007(3)$ & $-0.069(5)$ & $-0.032(4)$ \\
\hline$C(12)$ & $8 e$ & $0.0475(5)$ & $0.8393(4)$ & $0.1945(8)$ & $0.115(5)$ & $0.137(6)$ & $0.160(6)$ & $-0.043(4)$ & $0.006(5)$ & $-0.048(5)$ \\
\hline
\end{tabular}

Acknowledgments. The authors thank the Education Office of Hubei Province, P. R. China, for the research grant No. 2002B29002 and the Natural Science Foundation of Hubei Province, P. R. China, for the research grant No. 2003 ABB010.

\section{References}

1. Usman, A.; Fun, H.-K.; Zhu, H.-L.; Ke, G.: Bis(4-maleimidophenyl)methane. Acta Crystallogr. E59 (2003) o652-0653.
2. Sheldrick, G. M.: SHELXTL V5.1 Software Reference Manual, Bruker AXS Inc., Madison, Wisconsin, USA 1997. 